\title{
Abstraction of bio-inspired curved-line folding patterns for elastic foils and membranes in architecture
}

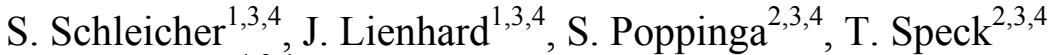 \\ \& J. Knippers ${ }^{1,3,4}$ \\ ${ }^{1}$ Institute of Building Structures and Structural Design (ITKE), \\ University of Stuttgart, Germany \\ ${ }^{2}$ Plant Biomechanics Group Freiburg, Botanic Garden, \\ Faculty of Biology, University of Freiburg, Germany \\ ${ }^{3}$ Competence Network Biomimetics, Baden-Württemberg, Germany \\ ${ }^{4}$ Bionics Competence Network (BIOKON e.V.), Germany
}

\begin{abstract}
Today's architectural foils and membranes amaze with their superior strength-toweight ratio and are often implemented as lightweight building envelopes or shading devices. Most claddings, however, are optimized for high tensile strength, which reduces the design possibilities to pre-stressed inflexible shapes. Only a few projects are exploring the potential inherent in the membrane's low bending stiffness. Nowadays, new materials and manufacturing methods allow for customized pliability of semi-rigid thin-shell structures, which fully tap the potential of reversible elastic deformation. While this concept has hardly been used in architecture, convertible surfaces are rampant in nature. Therefore, the aim of this paper is to review in general how nature's soft, flexible, and forceadaptive structures may inspire the development of technical membrane structures and outline their architectural potential in particular. Focusing on bio-inspired pliable systems that show distinct curved-line folding principles will be the framework for a close collaboration among architects, engineers, and biologists. Examining the flower opening of Ipomoea alba will clarify the drawbacks and opportunities of elastic kinematics. Therefore, the first part of the study will introduce this nocturnal flower, whose environmentally responsive petals adapt their geometry in a circadian rhythm. Morphological and anatomical analyses will secondly lead to a better understanding of their primarily
\end{abstract}


turgor-dependent cascade of multiple motion sequences. Examining the interaction of geometrically constraint surfaces and material-specific stress distribution in the flower's curved-line folding is thereby of particular interest. The plant's pattern will thirdly be abstracted and the interdependencies will be tested in digital models. Recording their packaging efficiency, mechanical simplicity, and structural characteristics will fourthly make the systems comparable. Finally, the project will deduce the physical principles by tracking the plant's kinematics and outline their use for architectural foils and membranes with similar adaptive behavior.

Keywords: plant movements, biomimetics, architecture, deployable structures, pliable structures, curved-line folding.

\section{Introduction}

In architecture, membranes and foils are valued for their lightweight and impressive tensile strength. These material characteristics allow for implementation as wide spanning structures or high performing envelopes. Only when forced in shape by tension, however, do they become structurally stable and can fulfill a predetermined function. As a result, the design possibilities of these structures are most often limited to pre-stressed inflexible shapes that avoid compression forces. Contrastingly, in many other fields of application in our daily life, we experience textiles and films as flexible and multifunctional structures. Used in garments, curtains, awnings, umbrellas or tents they respond to various user patterns, alternating structural loads, or changing environmental conditions. This changeability, however, comes at the expense of low load-bearing capacity and can only be attained with additional support structures.

Stability and flexibility within one structure and without the help of auxiliary constructions is a very challenging design goal. In architecture this is achieved mostly by structures that consist of rigid parts connected by local hinges. These hinges, however, are liable to attrition and are costly. An often neglected alternative to this approach is to balance both design goals with semi-soft or semi-rigid constructions whose structural versatility is based on differentiated material characteristics. Nowadays, manufacturing methods, such as tailoredfiber-placement or coating techniques, allow for customized pliability. By alternating a structure's stiffness distribution or superimposing additional material layers, for example, one can create folding areas that locally replace hinges or bracing zones that reinforce a structure as a whole. These design interventions enable the development of new foils and membranes, which fully tap the potential of reversible elastic deformations at the threshold to compression active thin-shell constructions.

\section{Abstraction of plant movements as concept generators for elastic mechanics in technical applications}

While the integration of ambivalent material characteristics within one design is a rather unfamiliar concept in the field of architecture, it is common in many 
living organisms. Plants, for example, show a broad spectrum of hinge-free movements with structures that provide flexibility while guaranteeing sufficient structural stability. An interdisciplinary collaboration among architects, engineers, and biologists revealed certain types of plant movements that can act as concept generators for deployable structures in architecture. The first examinations focused on reversible elastic deformations that can be observed in non-autonomous plant movements [1]; see also Poppinga et al. (this conference). Their deformation is generated by the application of a mechanical force on the respective structure (e.g. triggered by the weight of a pollinator). Further research exemplarily examined the valvular pollination mechanism in the flowers of Strelizia reginae and transferred their kinematics to a hinge-less versatile flap that was implemented into an adaptive façade shading system [2]; see also Lienhard et al. (this conference). This first project revealed that elastic kinematics are highly influenced by two main factors: geometry and material. Studying a plant's geometrical constraints, its force equilibrium, and its energy distribution is a necessary step for including autonomous plant movements into the screening process as well. Here, an active change of turgor pressure or cell wall swelling/shrinking drives the movement [3]. The interdependencies found between geometry and acting forces become even more relevant for a structure's deformation as soon as one combines multiple motion sequences together to a pliable system.

\section{Pliable systems}

Unlike other mechanical systems, which act mostly geometrically, the abstraction of pliable structures is more difficult as the physical behavior is enforced by a very complex interaction of geometrical stiffness and material stiffness. The resulting drawbacks and opportunities of these influencing factors can be explained exemplarily with curved-line folding. In contrast to straight-line folds where hinged surfaces transmit only axial forces, curved-line folds also distribute bending moments. Thus, bending a surface on one side of the fold is always accompanied by an associated bending of the surface on the other side. Both surfaces are coupled kinematically. The rules that govern in general a curved-line folding have already been identified with the help of theorems from differential geometry by Duncan and Duncan [4]. One result is that curved-line folds always separate convex and concave surfaces that have surface normal curvatures in the direction of the fold line of equal magnitude. Even though these kinematical studies can help predict a pattern's folding geometry, they describe only inextensible surfaces whose materiality is neglected. Pliable systems in nature, however, are not only kinematically constrained, but also informed by material characteristics. Moreover, different material properties within a curvedline structure highly affect the bending behavior, which makes the resulting deformation difficult to predict. Manipulating the shape or material set-up between pairs of surfaces, however, can also be functionalized and thereby gear a coupling with various transmission ratios. When balanced correctly, material choice, stiffness distribution, and fiber orientation can activate, regulate, or 
suppress the movement in pliable systems. Concerning this fine-tuning, biological case studies give valuable insights into possible geometrical and material adjustments. In this context, the authors started a first examination on the flower of Ipomoea alba, which fascinates not only by its interesting elastic opening mechanism but also by its distinctive curved-line folding pattern.

\section{Opening mechanism of Ipomoea alba (Convolvulaceae)}

Ipomoea alba, commonly known as white morning glory, moonflower or moon vine, is native to tropical and subtropical regions. The pentamerous, sympetalous funnel-shaped flowers show a circadian rhythm and open at dawn. As a characteristic for the Convolvulaceae, mid-petaline bands occupy the longitudinal middle third to fifth of each corolla segment [5]. In the opening process the densely packed and helically furled bud (fig.1A) pops open, and the flower lamina enfolds (fig.1B) within a few minutes. The flower's bud has a height of around $10 \mathrm{~cm}$ and a diameter of $1-2 \mathrm{~cm}$, which turns to a flower of $8-15 \mathrm{~cm}$ in diameter when blooming (fig. 1C). At dusk, the flowers close by rolling up, not resetting to their initial contort state (fig. 1D). Due to all these different movements and functional states the flower provides an excellent model for studying structural changes during maturation and senescence.

Deployability in the Ipomoea flower is most presumably enabled by three characters that highlight its specificity and make it an applicable role model for our project: 1) The corolla must be packed in a certain densely way to allow for the compact bud to turn into an exceptionally large flower. 2) Furthermore, this packaging must enable the fast and precise unfolding. 3) The role of coupled kinetics due to the flower's curved folding pattern in its opening and closing is completely unexplored up to now. First morphological and anatomical analyses of Ipomoea alba revealed some characteristic features during all its transitional states in which the flower has to balance a specific set of internal and external influencing factors. In the sympetalous flowers all petals are fused together to one continuous corolla surface. While other flowers can react by overlapping or sliding individual petals independently, Ipomoea has to address structural changes by folding, bending, and expending its corolla as well as by refining

(A)

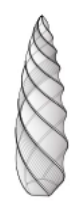

(B)

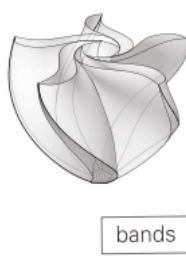

(C)

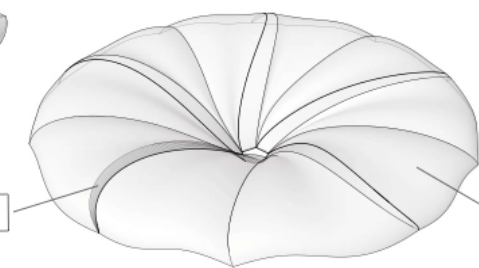

(D)

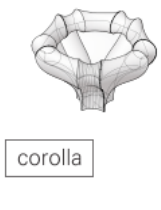

Figure 1: Transitional states of the Ipomoea alba flower. A: bud B: opening process C: open flower D: closed flower. 


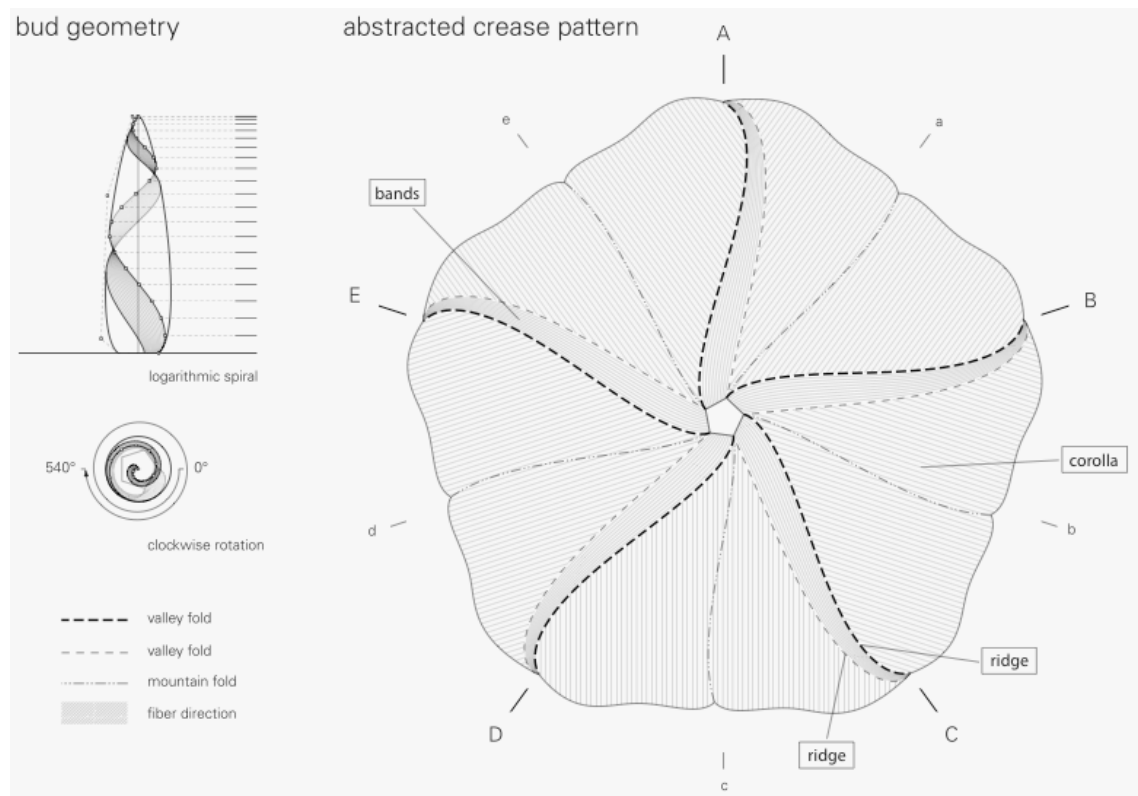

Figure 2: $\quad$ Morphology of Ipomoea alba in closed and open states.

locally and globally its stiffness distribution and the cell orientation of its tissue. Former anatomical analyses [6-9] identified the actuating elements responsible for the flower movement. On both ridges of the bands (fig. 2), cells show differences in expansion (in part due to turgor loss) and thus drive the unfolding und roll-up process.

In its bud-state the plant provides protection to its sexual organs with an efficient corolla packaging scheme (fig. 2). The relatively stiff mid-petaline bands are fully exposed to the exterior, whereas the rest of the corolla is internally folded. The five bands are wrapped by $540^{\circ}$ clockwise around a middle axis and form a logarithmical helix. The outer ridges of each band are adjacent to the ridges of its neighbor and thereby all bands together form a protective shell. At the beginning, the adjacent bands seem to be coadunate, only connected by a very thin lamina that seems to be a predetermined breaking region as soon as the bands start to separate.

The following opening mechanism is highly complex and can be considered as an interaction of two clearly distinguishable individual motion sequences. A spiral movement of the bands on the one side is accompanied by the unwrapping of the corolla on the other (fig. 1B). The plant's motion sequence is starting with the opening of the bud shell. After the thin lamina that connects the bands is torn apart the five bands start to separate from each other and bend outwards gently in helical movement. Even though little is known about the exact geometry of the bud's global transformation, the local structural changes that seem to drive the movement have been introduced. Phillips and Kende [10] examined the movement of the congener Ipomoea tricolor. In accordance to their results and to 
data of Poppinga et al. (this conference) it can be considered as passive autonomous nastic movement. They identified a specific group of inner epidermal cells near the band's ridges that act as the internal actuators by passively undergoing extensive vacuolation, while other cells in the tissue are already vacuolated. Just prior and during the flower opening this special cell group shows significant increase in size, which results in a surface extension of the band's inner tissue. Furthermore, the difference in the cellular turgor pressure inside and outside of the bands creates shear forces and thus is responsible for the plant's bending movement. Up to now, it is not clear if this mechanism is the only driving force or if there are other principles involved that might contribute to the flower opening. It is conceivable that the bands that are bent helically store elastic energy in their initial position. Once the bands separate from each other, this stored kinetic energy would release a force that drives a stress relaxation until each band has found another state of equilibrium. This 'burst' is known from other plants as 'bud-popping'. Besides the untwisting mid-petaline bands, the opening mechanism of Ipomoea alba shows another interesting motion sequence. While the bands fold outwards the petal corolla, which is previously furled up like an awning, unwraps and unfolds (fig. 1B). It is not clear if the corolla surface is just pulled by the attached bands following passively their deformation sequence, or if the corolla tissue takes part actively in the opening process by an autonomous deformation movement. This idea would raise some interesting questions and make the corolla opening an interesting field for further studies. In architectural applications like blinds and awnings, for example, architects are often confronted with similar challenging tasks, e.g. the rolling of double curved textiles to efficient packaging schemes. Unlike Ipomoea alba, rolled architectural surfaces do not have any folds or double layers that could possibly interfere with the rolling mechanism. Furthermore, the corolla of Ipomoea alba forms a doubly curved funnel-shaped surface when unrolled, while architectural blinds and awnings are up to now limited to structurally less stable uncurved deployable surfaces.

When blooming, the bud of Ipomoea alba turns into an impressive corolla with a diameter of up to $15 \mathrm{~cm}$, highly attractive to pollinating insects (fig. 1C). It demonstrates not only a very efficient packaging ratio but can rightly be called a large spanning structure. Furthermore, various interesting observations can be made when the flower is fully extended, which triggered the later described studies. Firstly, the blooming corolla forms a large cantilevering funnel that gains stability from its double curvature and a rolled brim. Thereby, the corolla is stable enough to carry the load of various pollinators like bumblebees or withstand smaller wind gusts. Secondly, the untwisted bands stretch the corolla wide open. Most noteworthy, however, is that each rib shows a clear S-shape in which the outer edge with the higher curvature seems to be much stiffer than its counterpart (fig. 2). Finally, the open flower reveals that the cell orientation in the band tissue is different than in the corolla lamina. While the fibers in the bands seem to follow the 'S-shape' of the ridges and are particularly distinctive in the region that was defined to drive the movement, the fibers in the corolla run parallel to the lamina crease, which presumably conforms to the rotation axis of 
its rolled-up state. Finally, during flower closing, the bands start curling inward by more than one complete turn and roll to a toroidal shape (fig. 1D). Similar to the opening mechanism, the same group of actuating cells seems affected by asymmetric turgor pressure and is controlling the closing mechanism [7].

\section{First kinematical study of Ipomoea alba}

In a first approach we examined the relationship between the geometry of the closed bud and the open corolla with its distinctive S-shaped bands. Therefore, we remodeled the bud in the digital environment of the $3 \mathrm{~d}$ design software Rhinoceros (fig. 3). Therein, the bud was abstracted as an ellipsoid by rotating a spline curve around a middle axis. This ellipsoidal surface provided a constant base for studying various band configurations with alternating rotation angles. The band's developable surface in its spatial helical form was compared with its simplified flat state. Two methods were chosen to generate individual sets of helices that rotate clockwise around the solid: Helices with a constant rotational angle and helices with a logarithmically increasing angle. The torsion of the helices graduates in steps of $90^{\circ}$ and ranges from $0^{\circ}-900^{\circ}$. In a next step each band was flattened and the radius of the resulting corolla was measured (fig. 4).

First results show that the corolla radius is dependent on the amount of turns in the helix. The more turns occur in the bud, the larger is the resulting size of the corolla (fig. 4). Thereby, helices with constant windings can generate larger corollas than logarithmical helices. Secondly, higher winding results in a more distinct S-shape of the bands. It is noteworthy that especially the helix with a logarithmically increasing rotation angle of $540^{\circ}$ resembles the bands of Ipomoea alba the most. Finally, this study shows that the S-shape of the ridges is most likely a pure geometrical factor and might by itself only play a minor role in the opening mechanism. To what extent, however, the S-shape geometry interacts with the flower's irregular stiffness distribution and its specific material characteristics as part of a pliable system remains yet unsolved and was the motivation for the next step of kinetic analyses.

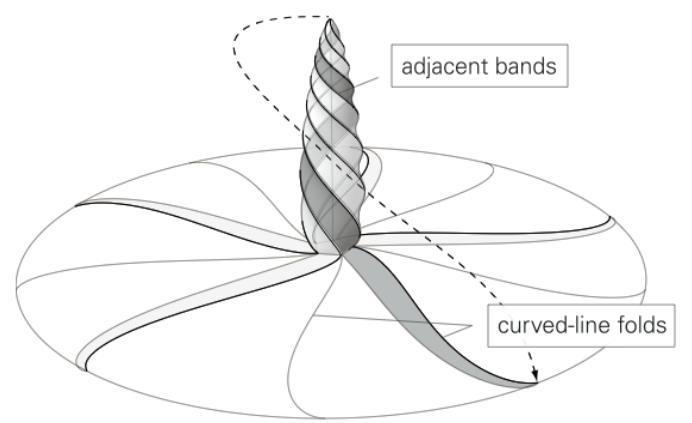

Figure 3: $\quad$ Abstracted digital model of an Ipomoea alba flower. 


\section{Helices with constant rotation angle}
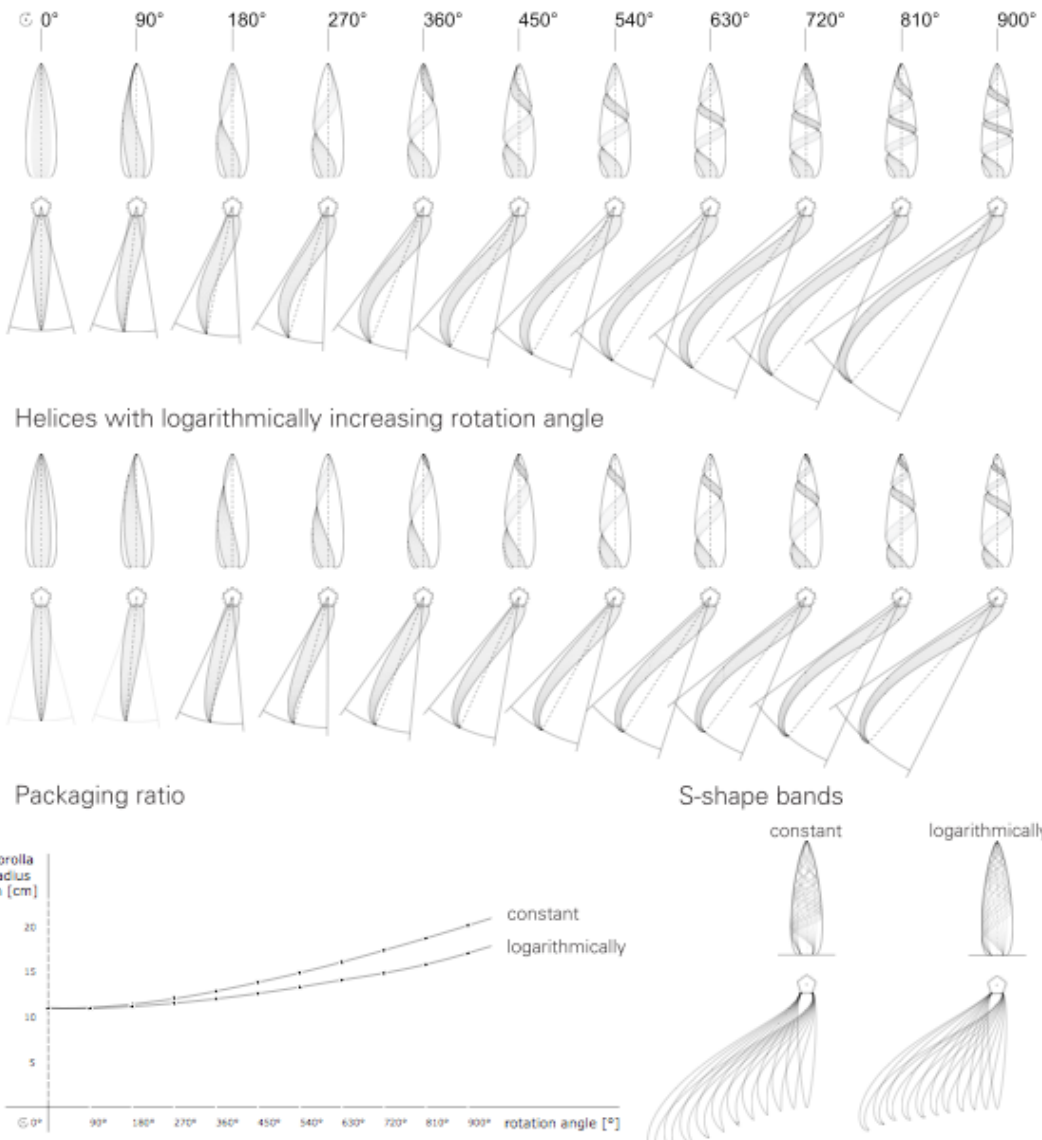

S-shape bands
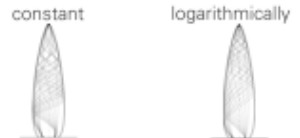

Figure 4: $\quad$ Helices with constant and logarithmical rotation angle.

\section{Informed kinetics}

In order to investigate the interaction between the flower's geometry and its materials, we transferred our digital model to the finite element software Sofistik. Firstly, we compared five bands with different shapes (fig. 5): strips of rectangular (A), triangular (B), curved triangular (C), and S-shaped (D) form. In addition, we added a strip that is formed like a band of Ipomoea alba with one stiff ridge (E). Secondly, we simulated the plants cells extension by applying a difference in temperature to the top and underside of the shell elements. Each band was supported statically determinate and was thus unrestrained in its temperature driven deformation. As expected the bands start curling with increasing difference in temperature. Interestingly, the bending radius of the 

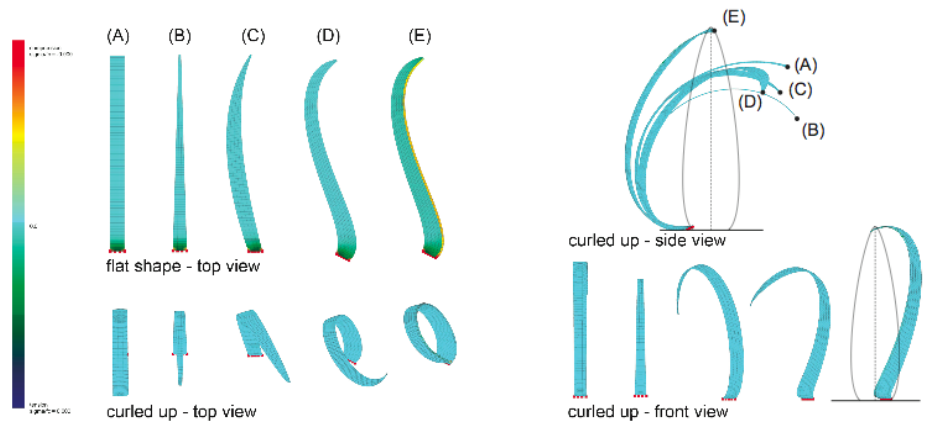

Figure 5: Force-driven bending.

Tracking curling paths of all bands (side view)

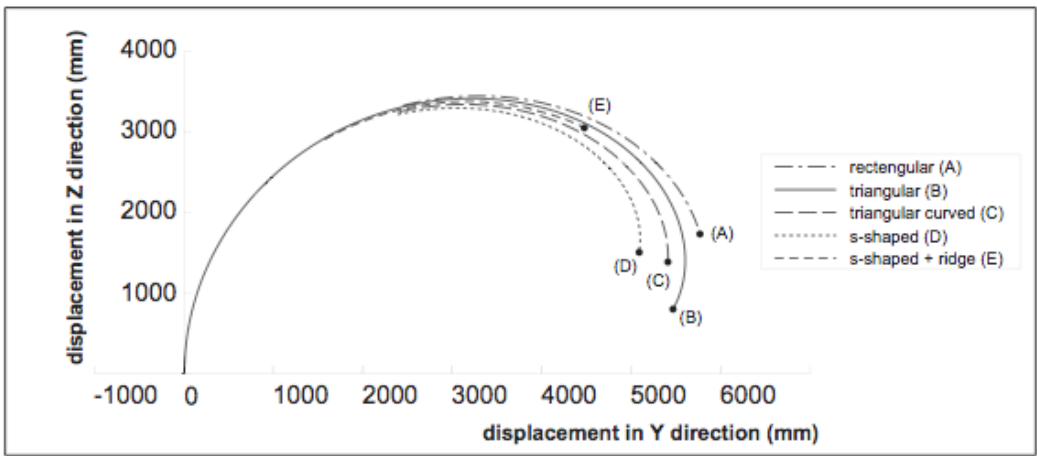

Tracking curling paths of s-shaped bands (top view)

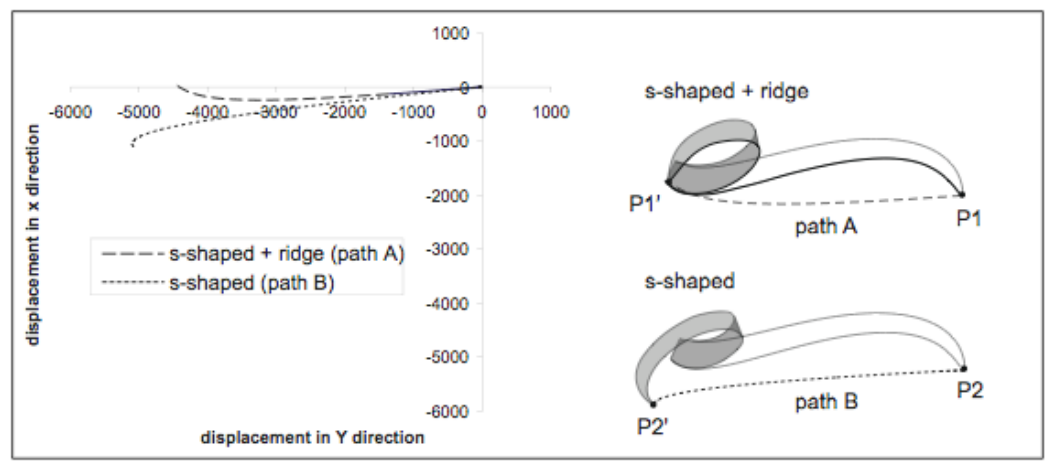

Figure 6: Tracking of curling paths. 
triangular bands is smaller than that of the rectangular (fig. 5 side view). The Sshape forms, however, curl the most. This was also observed in the biological model. With the help of the finite element simulation the equilibrium paths of the tip of each band was traced (fig.6). In the side view one can see that the paths of the five tested band geometries almost match until they reach their highest point. When the curling starts, the tips of the bands sink down and the equilibrium paths are starting to differ considerably. Here it can be seen that version (E) with its stiff ridge gains the most height and thereby stays the closest to the vertical axis. In the top view it can be noticed that the initially expected curling around the vertical axis of the S-shaped bands does not occur. This may underline the assumption that the opening of Ipomoea alba consists of two sequences, where curling around the vertical axis only occurs in the first motion sequence, which is initiated by the bud popping. This vertical curling is irreversible as it occurs due to diminution of internal mechanical tension. The second sequence is theoretically reversible and matches the results of the finite element simulation. These initial tests therefore suggest the conclusion that the stiff curved-line ridge does play a role in the opening mechanism of Ipomoea alba.

\section{Conclusion}

Ipomoea alba demonstrates new concepts for deployable structures. Its unfolding and closing principles hold a high potential for developing the next generation of umbrellas and awnings. First tests simulate and predict mechanics that are no longer based on hinged solids but use elasticity as a basis for optimized deployable structures. Of course, further studies are needed to understand the precise interaction between material stiffness and geometrical constraints and to inform an optimized balance. The presented preliminary studies, however, help to conceive the set-ups of further structural tests and analyses of the plant's construction details. It is conceivable that polymorphous foils and membranes are suitable materials for technically transferring the deformation behavior of biological models like Ipomoea alba.

\section{Acknowledgements}

This research is part of the joint research project 'Deployable structures in architecture - flexible surface structures on the basis of bionic principles', which is supported within the funding directive BIONA by the German Federal Ministry of Education and Research. Additionally, the German National Academic Foundation supports the first author's dissertation. Finally, we would like to thank our project partners for many helpful discussions.

\section{References}

[1] Matini, M. R., Knippers, J., Application of "abstract formal patterns" for translating natural principles into the design of new deployable structures in 
architecture. In: Design and Nature IV, WIT Press, Southampton, ed. C.A. Brebbia, pp. 147-156, 2008.

[2] Lienhard, J., Poppinga, S., Schleicher, S., Masselter, T., Speck, T., Knippers, J., Abstraction of plant movements for deployable structures in architecture. Proc. of the 6th Plant Biomechanics Conf., ed. B. Thibaut, Cayenne, French Guyana, pp. 389-297, 2009.

[3] Burgert, I., Fratzl, P., Actuation systems in plants as prototypes for bioinspired devices. Philosophical Transactions of the Royal Society A 367, pp. 1541-1557, 2009.

[4] Duncan, J.P., Duncan, J.L., Folded Developables, Proceedings of the Royal Society of London, Series A, Mathematical and Physical Sciences 383, 1784, pp 191-205, 1982.

[5] Heywood, V.H., Brummitt, R.K., Culham, A., Seberg, O., Flowering Plant Families of the World. Royal Botanic Gardens, Kew, London, 2007.

[6] Kende, H., Baumgartner, B., Regulation of Ageing in Flowers of Ipomoea tricolor by Ethylene, Planta 116, pp. 279-289, 1974.

[7] Hanson, A. D., Kende, H., Ethylene-Enhanced Ion and Sucrose Efflux in Morning Glory Flower Tissue, Plant Physiol. 55, pp. 663-669, 1975.

[8] Kaihara, S., Takimoto, A., Effects of light and temperature on floweropening in Pharbitis nil. Plant and Cell Physiology 22, pp. 215-221, 1981.

[9] Doorn W. G., Meeteren U., Flower opening and closure: a review. Experimental Botany 54, 389, pp.1801-1812, 2003.

[10] Phillips, H.L., Kende H., Structural changes in flowers of Ipomoea tricolor during flower opening and closing, Protoplasma 102, pp. 199-216, 1980. 